RESEARCH ARTICLE

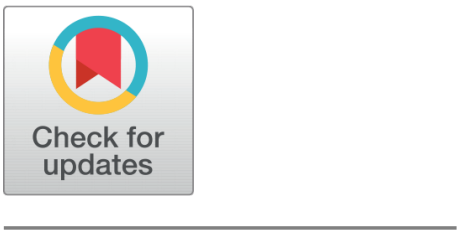

OPEN ACCESS

Received: 09.02.2021

Accepted: 03.03.2021

Published: 15.03.2021

Citation: Pareek D, Purohit P (2021) Transit of the Sun across constellation Libra and variation of secondary gamma radiation flux in the month of October and November, 2020 at Udaipur, India. Indian Journal of Science and Technology 14(8): 718-724. https://d oi.org/10.17485/IJST/v14i8.263

* Corresponding author.

Tel: 9413954886

deven.pareek69@gmail.com

Funding: None

Competing Interests: None

Copyright: @ 2021 Pareek \& Purohit. This is an open access article distributed under the terms of the Creative Commons Attribution License, which permits unrestricted use, distribution, and reproduction in any medium, provided the original author and source are credited.

Published By Indian Society for Education and Environment (iSee)

ISSN

Print: 0974-6846

Electronic: 0974-5645

\section{Transit of the Sun across constellation Libra and variation of secondary gamma radiation flux in the month of October and November, 2020 at Udaipur, India}

\author{
Devendra Pareek $^{1 *}$, Prajesh Purohit ${ }^{1}$ \\ 1 Department of Physics, Bhupal Nobles' University, Udaipur, 313001, India. Tel.: 9413954886
}

\section{Abstract}

Background: During transit of the Sun across the constellation Libra in the month of October and November 2020 we experimentally observed the variation of secondary gamma radiation flux (SGR) at Udaipur (270 43' 12.00" $\mathrm{N}, 75028^{\prime} 48.01 " \mathrm{E}$ ), India. We interpret such variation of SGR flux on the basis of gravitational lensing effect produced by the Sun and constellation Libra, radiation from constellation and gravitational pull-on background radiation by constellation Libra and Sun. Objective: The study is to observe secondary gamma radiation flux during transit of the Sun across the constellation Libra. Method: For this experimental study we used ground based Nal (TI) Scintillation detector. Data were collected around time from 17.0 IST to 17.30 IST on date October 27, 28, 29, 30, 31 and November 2, 4, 6, 11, 12, 13, 14, 2020. The detector was pointed towards the Sun in this whole experimental study. Finding: Analyzed data showed significant variation of secondary gamma radiation flux (SGR) in month of October and November. Novelty: Due to transit of the Sun across the constellation Libra on the surface of Earth secondary gamma radiation flux varies. In the Month of October from 27 October to 31 October secondary gamma radiation flux decreases from 240084 to 230424 while in the month of November from November 2 onward dates up to 12 November the secondary radiation flux increases from 234414 to 242816 and then started to decrease on date 13, 14 November 2020.

Keywords: Cosmic radiation; solar radiation; secondary gamma radiation; the Sun; Libra constellation; gravitational pull; radiation from constellation; gravitational lensing effect

\section{Introduction}

Cosmic radiation travels at nearly the speed of light and it is compos of about $89 \%$ protons, $10 \%$ of helium, and about $1 \%$ of others heavier elements ${ }^{(1-3)}$. This type of radiation propagates through space, arrives on the Earth. Energy range of primary cosmic radiation is from $10^{9} \mathrm{eV}-10^{20} \mathrm{eV}$ or more ${ }^{(4)}$. Radiation coming from the Sun is known as solar radiation (SR). Simpson, J. (1983) ${ }^{(5)}$ pointed out chemical abundances 
of cosmic radiation in different energy range. Cosmic radiation (CR) and solar radiation (SR) strikes on atoms of the atmosphere of the Earth produce secondary particles known as secondary radiation ${ }^{(6)}$. Therefore, penetrating radiation produced secondary shower ${ }^{(7)}$. Particles increase rapidly as these move downward in the atmosphere and in each interaction the particles loose energy ${ }^{(8,9)}$. Secondary particles have pions, muons, neutrinos gamma radiation, electrons and positrons. In this way secondary particles shower down through the atmosphere to the Earth's surface ${ }^{(10)}$. Such radiation has three components as electromagnetic components, hadronic component and masonic component ${ }^{(11-13)}$.

Gamma radiation in secondary particles known as secondary gamma radiation (SGR) flux. At surface of the Earth secondary radiation can be detected using appropriate detector ${ }^{(14,15)}$.

A. S. Eddington and collaborators in a famous experiment during a total solar eclipse in 1919 observed the gravitational lensing effect due to Sun. Electromagnetic radiation bends by the massive objects when passing near the object. It is due to gravitational field of the object ${ }^{(16-18)}$. The object could be a Star, galaxy or a cluster of galaxies.

During solar eclipses various researches ${ }^{(19-23)}$ observed the variation of radiation flux.

In ${ }^{(24)}$ observed variation in secondary gamma radiation flux during lunar eclipse. It can be explained on the basis of bending of primary cosmic radiation and solar radiation due to combine gravitational lensing effect of Sun and Earth and back scattered Secondary flux form the Moon.

Using Scintillation counter system ${ }^{(25)}$ did experimental of phases of moon in the month of September 2000 to observe secondary radiation flux. This experimental study had been planned to observe gravitational lensing effect. On September 9 and 10, 2000Moon passes background of Capricornus constellation and on these dates, we observed change in energy spectra of secondary gamma radiation due to gravitational lensing effect.

With the fact that during different celestial events happening in sky, modulate flux of cosmic and solar radiation we conducted ground based experimental study of transit of Sun across constellation Libra using scintillation detector at Udaipur, India.

\section{Experimental setup and observations}

The Scintillation detector of Nucleonix make (SD 152 F) flat type (Figure 1) was used to detect the secondary gamma radiation flux. Size of $\mathrm{NaI}(\mathrm{Tl})$ crystal is 2 " x 2" and optically coupled with photo multiplier tube. This set up was connected to $1 \mathrm{k}$ multichannel analyzer (MC 1000 with 1024 channel) of Nucleonix make with usb interface built in high voltage and shaping amplifier. For this experimental study the Scintillation counter system kept open to collect the counts as a function of time on the roof of Astronomy Laboratory of Department of Physics, Bhupal Nobles' University Udaipur (270 43' 12.00” N, 750 28' 48.01” E) (Rajasthan) India. Data were collected around time from 17.0 IST to 17.30 IST on date October 27, 28, 29, 30, 31 and November $2,4,6,11,12,13,14$. The detector was pointed towards Sun in this whole experimental study.

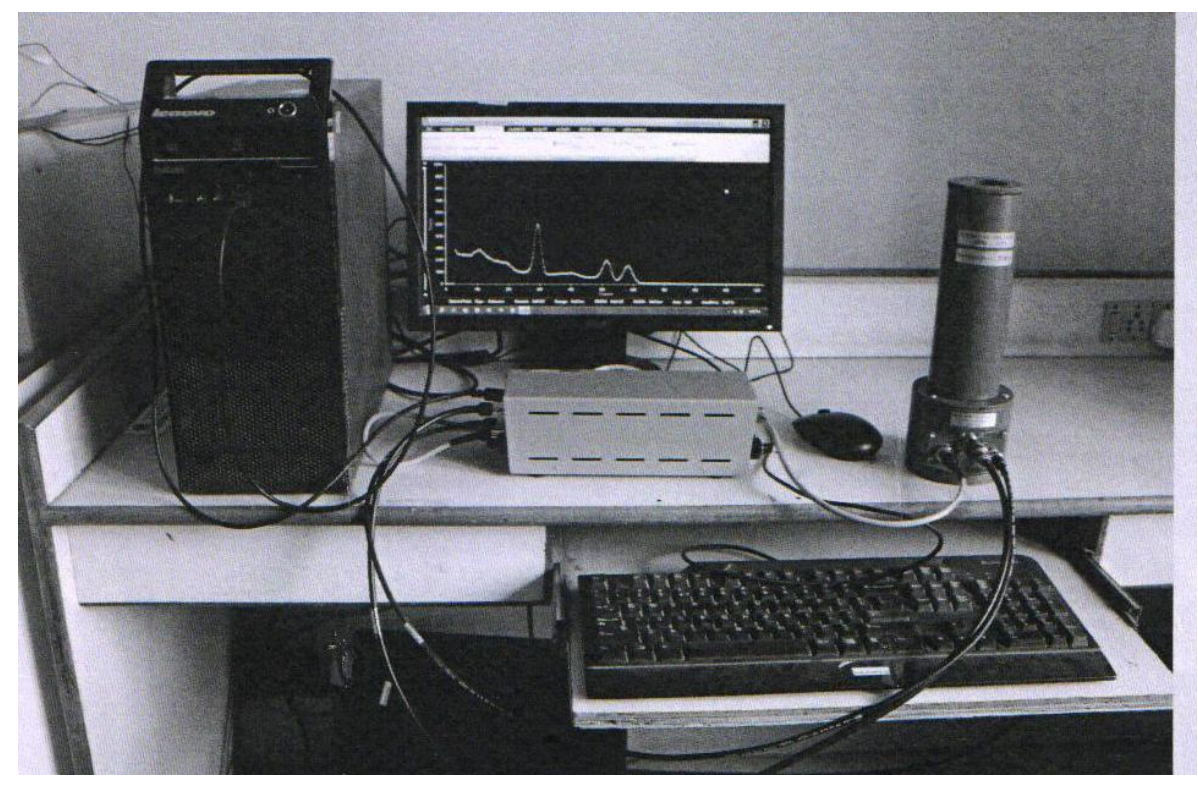

Fig 1. Scintillation Counter System 


\section{Results}

We started observation from October 27. The dates of observation were October 27, 28, 29, 30, 31 and November 2, 4, 6, 11, 12, 13, 14, 2020.

As depicted in Figure 2 the panels of SGR flux integrated data files between channel and dates of month October were taken around time from 17.0 IST to 17.30 IST.
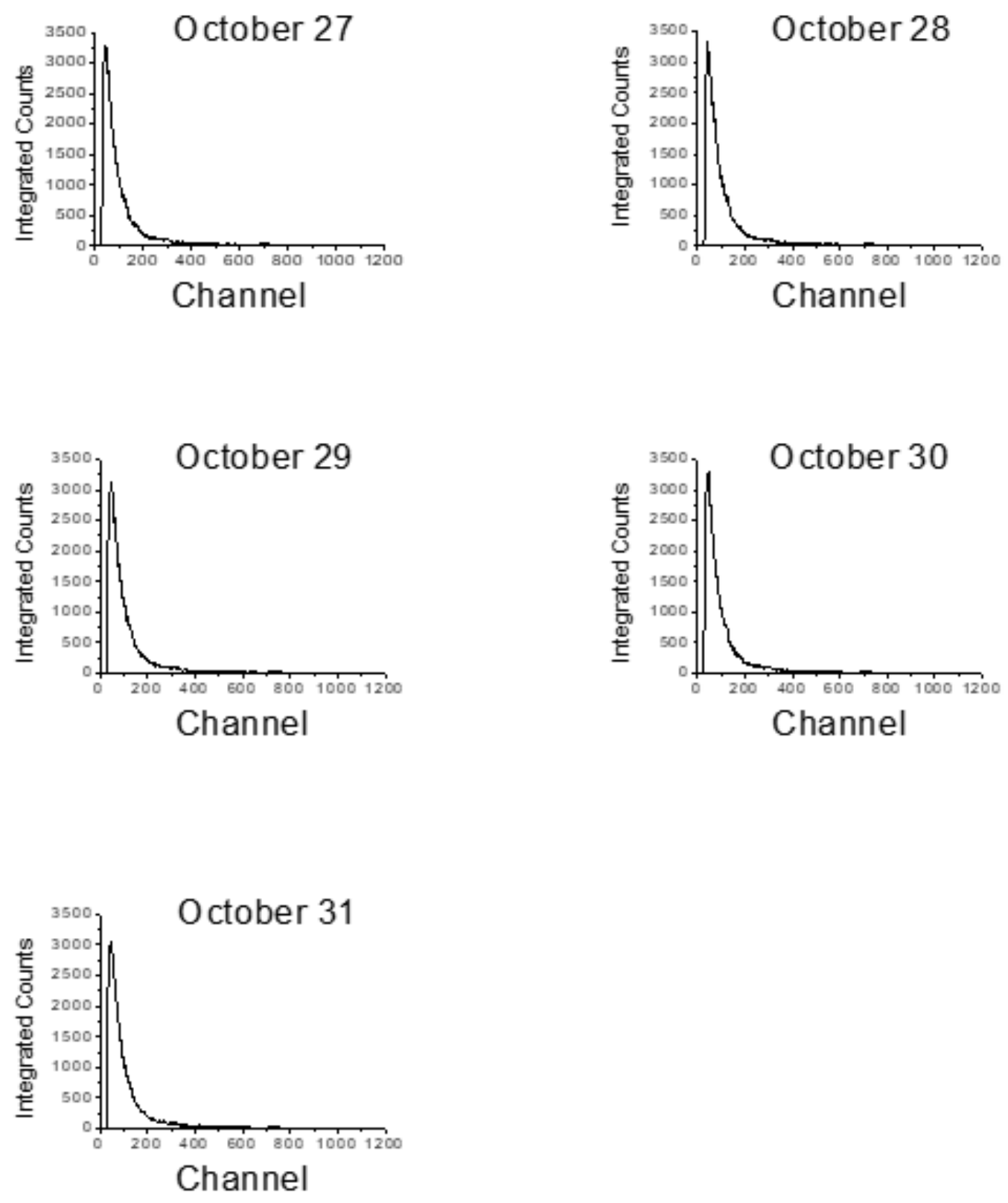

Fig 2. Panels of SGR flux integrated data files 
Also, Figure 3 represents for the month of November the panels of SGR flux integrated data files between channel and dates were taken around time from 17.0 IST to 17.30 IST.
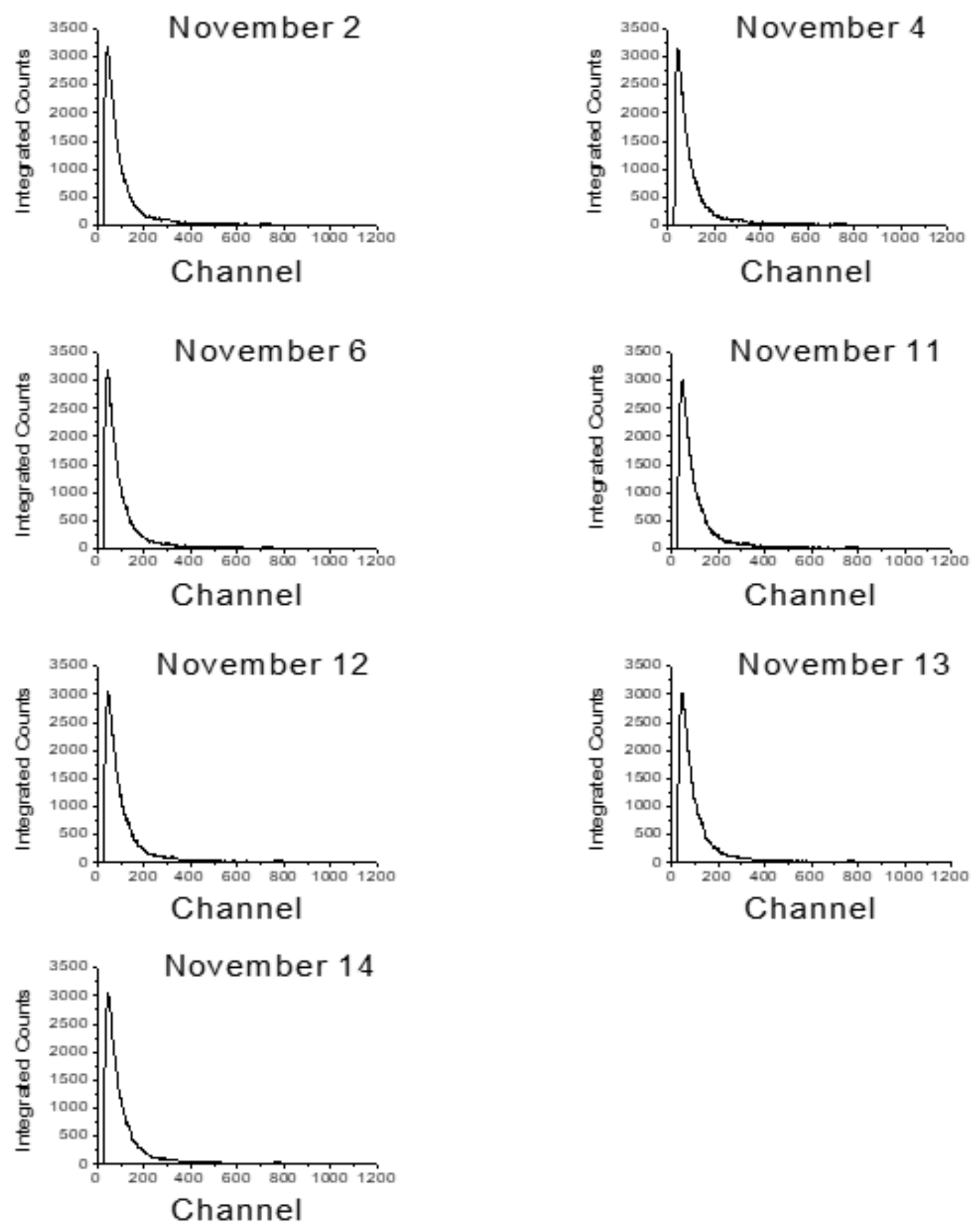

Fig 3. Panels of SGR flux integrated data files 
Using above panels of SGR flux integrated data files, we made Figures 4 and 5 which represent integrated counts of secondary gamma radiation flux with respect to dates in month of October and November 2020.

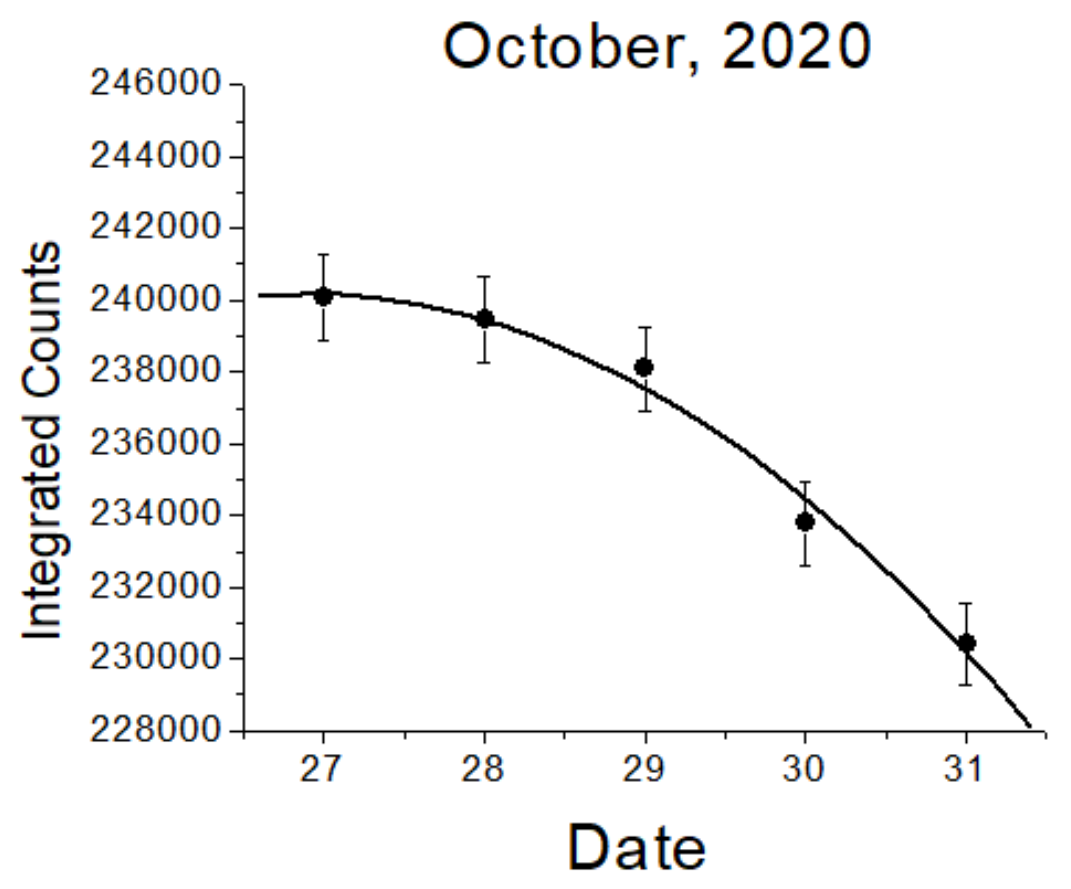

Fig 4. Integrated counts of secondary gamma radiation flux

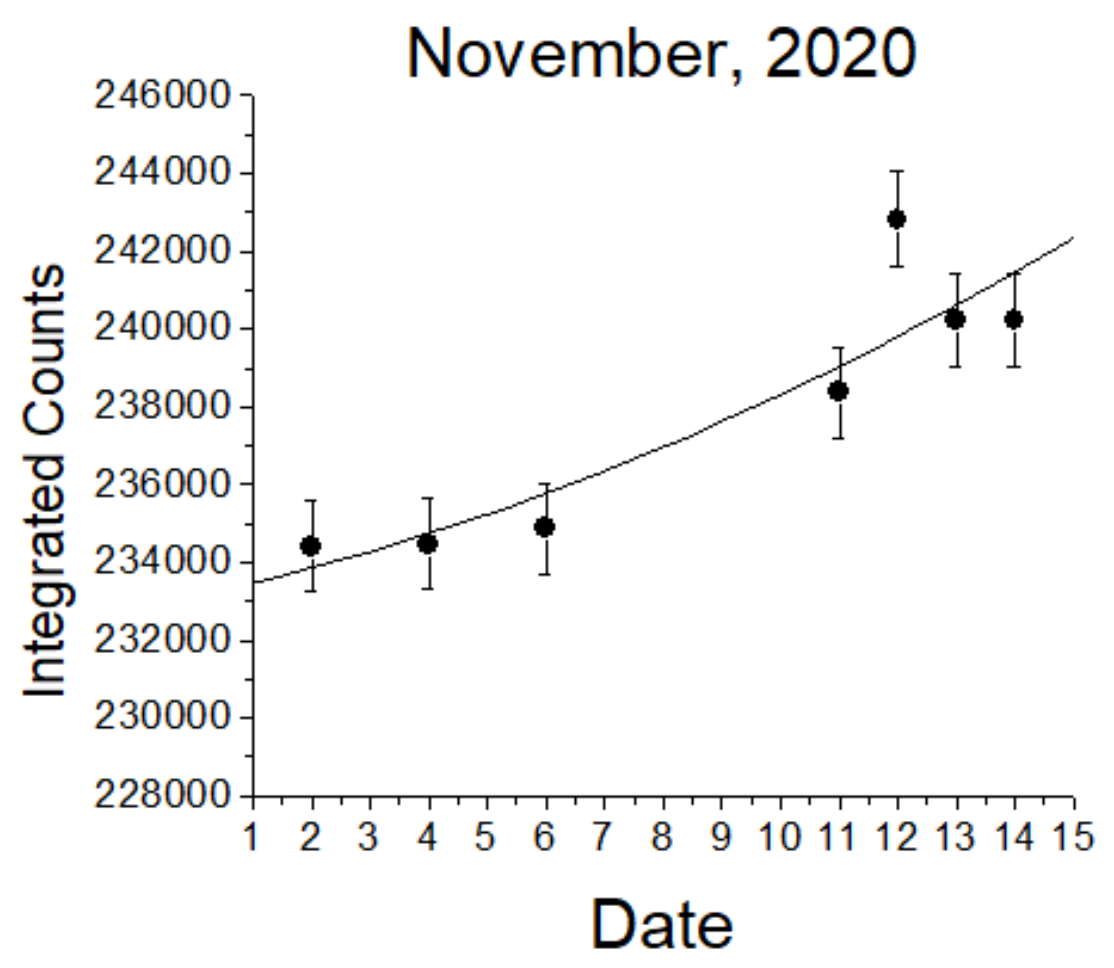

Fig 5. Integrated counts of secondary gamma radiation flux 
We made combine Figure 6 for the month of October and November between date and integrated counts to represent clear variation of secondary gamma radiation flux:

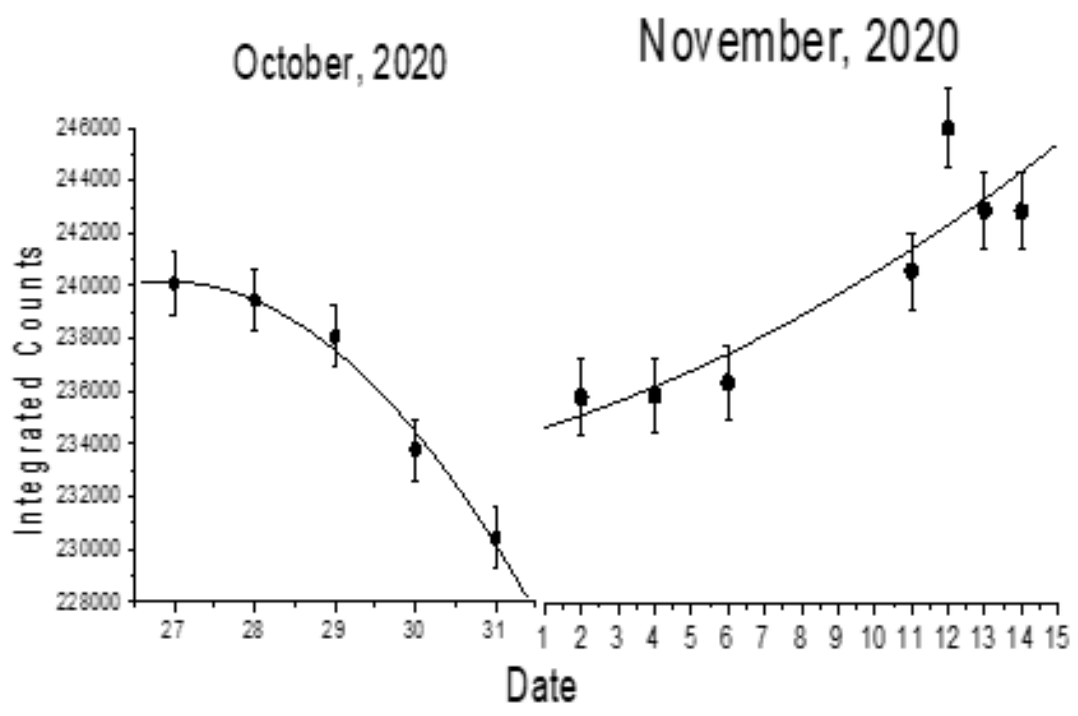

Fig 6. Integrated counts of secondary gamma radiation flux

Figure 6 represents that between the dates 27 October to 31 October secondary gamma radiation flux decreases from 240084 to 230424 . From November 2 onward dates up to 12 November the secondary radiation flux increases from 234414 to 242816 and then started to decrease on date 13, 14 November 2020. From date 27 October to 31 October the Sun was shifted away from the constellation Virgo. From November 2 the sun was approaching towards Libra constellation. The Sun was in the Libra constellation on date November 12, and we observed the highest counts. After this date the Sun started to shift away from Libra constellation and we observed less secondary gamma radiation flux on dates 13, 14 November.

\section{Discussions}

1. From Figure 6 we conclude that from 27 to 31 October onward integrated counts decreases because the sun was moved away from Virgo constellation. Therefore, we observed decreasing counts.

2. From November 2 the Sun was approaching towards Libra Constellation. On date November 12 the Sun was in the constellation Libra, and we observed the highest counts in this experimental study. The probable reasons are radiation from constellation, combine gravitational pull and gravitational lensing effect by the Sun and constellation on background radiation. Therefore, more radiation pulled and bent which may produces more shower of secondary gamma radiation particles in the atmosphere of the Earth.

3. After November 12 the Sun was shifted away from the constellation, and we observed fewer counts on comparison to November 12.

\section{Conclusion}

Variation of secondary gamma radiation flux during transit of Sun across constellation Libra is another signature. From November 2 the Sun was approaching towards Libra Constellation. On date November 12 the Sun was in the constellation Libra, and we observed the highest counts in this study as shown in Figure 6. Therefore, this study confirms the effect on secondary gamma radiation flux at the surface of the earth during transit of the Sun across constellation Libra. Using results of 
this study one can plan to observe variation of secondary radiation flux at the surface of the earth during transit of Sun across different constellations. Thus, this study helps to understand the variation of radiation flux during such astronomical events due to the combined gravitational lensing effect and gravitational pulling effect on the background radiation due to Constellations and Sun and radiation from constellations.

\section{Acknowledgements}

The authors are thankful to the students Kamlesh Audichya and Himanshu Singh Chouhan who were involved in this experimental study.

\section{References}

1) Longair MS. High energy Astrophysics;vol. 1. 2nd ed. and others, editor;Cambridge University press. 1992.

2) Chaisson E, Mcmillan S. Astronmy Today. 3rd ed. and others, editor;Prentice Hall. 1999.

3) Mewaldt RA. Cosmic rays. and others, editor;California institute of technology. 2010. Available from: http://www.srl.caltech.edu/personnel/dick/cos_ encyc.html.

4) Kudela K. On energetic particles in space. Acta Physica Slovaca Reviews and Tutorials. 2009;59(5):537-652. Available from: https://dx.doi.org/10.2478/ v10155-010-0098-4.

5) Simpson J. Elemental and isotopic composition of galactic cosmic rays. 1983.

6) Anderson CD, Neddermeyer SH. Cloud Chamber Observations of Cosmic Rays at 4300 Meters Elevation and Near Sea-Level. Physical Review. 1936;50(4):263-271. Available from: https://dx.doi.org/10.1103/physrev.50.263.

7) Heitler WH. On the analysis of cosmic rays. In: and others, editor. Proceeding of the Royal Society;vol. 161. 1937. Available from: https://doi.org/10. 1098/rspa.1937.0145.

8) Bhabha HJ. Nuclear Forces, Heavy Electrons and the $\beta$-Decay. In: and others, editor. Proc.Roy.Soc.Lond.A;vol. 166. Springer Science and Business Media LLC. 1938. Available from: https://dx.doi.org/10.1038/141117a0.

9) Bhabha. Nuclear forces, on the theory of heavy electrons and nuclear forces. Nature. 1938;141:117. Available from: https://doi.org/10.1038/141117a.

10) Allkofer OC, Grieder PKF. Cosmic Rays on Earth. . Available from: https://inis.iaea.org/search/search.aspx?orig_q=RN:15054748.

11) Heitler W. Showers produced by the penetrating cosmic radiation. Royal Society. 1938;166(927):529-543.

12) Nordheim LW. On the Absorption of Cosmic-Ray Electrons in the Atmosphere. Physical Review. 1937;51.

13) Pfotzer. Messungen der Ultrastrahlung in der Stratosphäremiteiner Dreifachkoinzidenzapparatur. Z Phys. 1936;102(23):41.

14) Kodama M. Ground Albedo Neutrons Produced by Cosmic Radiations. Physical Society of Japan, Journal. 1983;52:1503-1504.

15) Chilingarian A, Daryan A, Arakelyan K, Hovhannisyan A, Mailyan B, Melkumyan L, et al. Ground-based observations of thunderstorm-correlated fluxes of high-energy electrons, gamma rays, and neutrons. Physical Review D. 2010;82(4). Available from: https://dx.doi.org/10.1103/physrevd.82.043009.

16) Walsh D, et al. Nature. 1979;279(5712):381-384.

17) Mellier Y. Porbing the universe with weak lensing. 1998. Available from: https://arxiv.org/abs/astro-ph/9812172.

18) Narayan R, Bartelmann M. 1996. Available from: https://arxiv.org/abs/astro-ph/9606001.

19) Bhattacharyya A, Biswas S, Barun K, Chatterjee M, Das PK, Das TK, et al. Bikash Sinha \& Debapriyo Syam, Variation of $\gamma$-Ray and particle Fluxes at the Sea Level During the Total Solar Eclipse of. Astrophysics and Space Science. 1995;250:313-326.

20) Kandemir G. The Last Total Solar Eclipse of the Millennium in Turkey. ASP Conference Series. 2000;205.

21) Nayak PK, Gupta SK, Jain A, Mazumdar I, Raha S, Saha SK, et al. A study of the $\gamma$-ray flux during the total solar eclipse of 1 August 2008 at Novosibirsk, Russia. Astroparticle Physics. 2010;32(6):286-293. Available from: https://dx.doi.org/10.1016/j.astropartphys.2009.09.006.

22) Bhaskara A, Purohit A, Hemalatha M, Pai C, Raghav A, Gurada C, et al. Anirudha Patankar, A study of secondary cosmic ray flux variation during the annular eclipse of 15. Astroparticle Physics. 2010;35(5):223-229.

23) Pareek D, Jaaffrey SNA, Talesra KP, Yadav R, Ameta S. Experimental study of Variation of Secondary Cosmic Gamma Ray Flux and Energy during Partial Solar Eclipse of 4th January 2011 at Udaipur, India. Research journal of physical sciences. 2011;1(5):22-30.

24) Pareek D, Jaaffrey SNA. Experimental Study of Variation of Secondary Cosmic Gamma Ray Flux during Total Lunar Eclipse April 4, 1996 and July 16, 2000. Research journal of physical sciences. 1996;1(4):22-27.

25) Pareek D, Jaaffrey SNA. Experimental Study of Phases of Moon for observing Variation of Secondary Cosmic Gamma Ray Flux, Energy and X-Ray Flux in the Month of September. International journal of scientific research. 2000;3(5):6-10. 